\title{
Assembly of 12-tungstovanadate-templated nanocage and nanocomposites with single-walled carbon nanotubes as anodes in lithium-ion batteries
}

\author{
Jingquan Sha, ${ }^{\dagger} \dagger$ Jingwen Sun, $\stackrel{\perp}{ }$ Zhibo Tong, ${ }^{\dagger}$ Xiao Li,,${ }^{\dagger}$ Mengting Li,,$*$ Mingqi $\mathrm{Xu},{ }^{\dagger}$ \\ Qian Li, ${ }^{\dagger}$ Chang Liu ${ }^{\dagger}$ \\ $\uparrow$ Key Laboratory of Inorganic Chemistry, Department of Chemistry and Chemical \\ Engineering, Jining University, Qufu, Shandong, 273155, P.R China. \\ $\$$ School of Chemistry and Chemical Engineering, Qufu Normal University, Qufu, \\ Shandong 273165, P.R China. \\ ${ }^{\perp}$ School of Pharmacy, Qiqihar Medical University, Qiqihar, 161006, P. R. China
}

\section{Corresponding Author}

Jingquan Sha, E-mail: shajq2002@126.com

Mengting Li, E-mail: limt0205@qfnu.edu.cn 


\section{Experimental and synthetic details}

\section{Materials and Characterization.}

All reagents were commercially purchased from Alfa Aesar Company and used without further purification. Single-walled carbon nanotubes (SWNTs, purity $>95 \%$ ) were purchased from Chengdu Organic Chemicals Co. Ltd, Chinese Science Academy. The elemental analyses were measured by the Perkin-Elmer $2400 \mathrm{CHN}$ elemental analyzer for $\mathrm{C}, \mathrm{H}$, and $\mathrm{N}$ and Agilent ICPOES730 for Ag, V, W. The FT-IR spectra were obtained from the Alpha Centaurt FT/IR spectrometer with $\mathrm{KBr}$ pellets. The power X-ray diffraction (PXRD) patterns were scanned by the Rigaku D/MAX $2500 \mathrm{~V}$ XRD diffractometer with $\mathrm{Cu}-\mathrm{K} \alpha$ radiation. Scanning electron microscopy (SEM) images were obtained using a Zeiss Supra 55 SEM. Raman spectra were measured with a Renishaw micro-Raman spectrometer using $633 \mathrm{~nm}$ argon-ion laser as excitation radiation source. The morphology and energy dispersive X-ray (EDX) analysis were operated on a scanning electron microscopy (SEM, Phenom ProX) with an accelerating voltage of $15 \mathrm{kV}$. Transmission electron microscopy (TEM) on JEM-200CX apparatus was performed at an accelerating voltage of $200 \mathrm{kV}$.

\section{Preparation of the $V W_{12} @ M O C F$ nanocrystals}

Crystal of VW $12 @$ MOCF (150 mg) were ground for 3 h by chemical ball milling, then the resulting powder was dissolved in methanol $(40 \mathrm{~mL})$ and sealed in a Teflon antoclave, which was heated in a microwave reactor at $300 \mathrm{~W}$ for $4 \mathrm{~h}$. The resulting VW $12 @$ MOCF nanocrystals were separated by centrifugation, rinsed with water, dried in a vacuum at $80^{\circ} \mathrm{C}$ for $24 \mathrm{~h}$ (yield: $121 \mathrm{mg}$ )

\section{Synthesis of $\left(\mathrm{n}-\mathrm{Bu}_{4} \mathrm{~N}\right)_{3}\left[\mathrm{VW}_{12} \mathrm{O}_{40}\right]$}

The $\left(\mathrm{n}-\mathrm{Bu}_{4} \mathrm{~N}\right)_{3}\left[\mathrm{VW}_{12} \mathrm{O}_{40}\right]$ was synthesized according to the previous report. Typically, to a solution of $8.3 \mathrm{~g} \mathrm{Na}_{2} \mathrm{WO}_{4} \cdot 2 \mathrm{H}_{2} \mathrm{O}$ and $2.9 \mathrm{~g} \mathrm{NH}_{4} \mathrm{VO}_{3}$ in $35 \mathrm{ml}$ of warm water was added $400 \mathrm{ml}$ of $\mathrm{CH}_{3} \mathrm{CN}$. To the mixture consisting of two liquid-layers was added dropwise $65 \mathrm{ml}$ of conc. $\mathrm{HCl}$ with continuous stirring. The resultant turbid solution was heated at $70{ }^{\circ} \mathrm{C}$ for $24 \mathrm{~h}$. After cooling to room temperature, the precipitate was filtered off. To the filtrate was added $15 \mathrm{~g}$ of $\mathrm{n}-\mathrm{Bu}_{4} \mathrm{NBr}$ to precipitate a white salt. The salt was collected by filtration, and washed with water and ethanol, and air-dried 
(yield: 1.98g). Found: V, 1.36; W, 60.4\%.Calc. for $\left(\mathrm{n}-\mathrm{Bu}_{4} \mathrm{~N}\right)_{3}\left[\mathrm{VW}_{12} \mathrm{O}_{40}\right]: \mathrm{V}, 1.41 ; \mathrm{W}$, 60.9\%. IR (solid KBr pellet/cm-1): 976(s), 892(s) and 783(s).

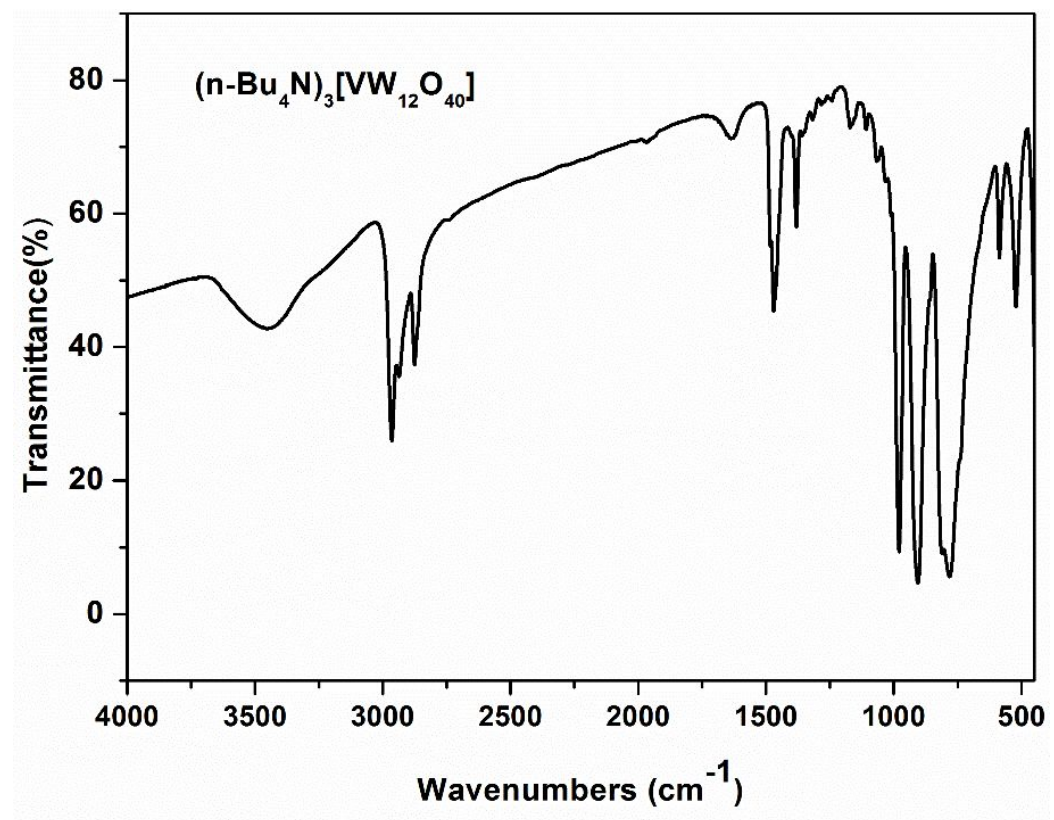

Figure S1. The IR spectrum of compound $\left(\mathrm{n}-\mathrm{Bu}_{4} \mathrm{~N}\right)_{3}\left[\mathrm{VW}_{12} \mathrm{O}_{40}\right]$. The characteristic vibration modes of the Keggin POMs at 976, 892 and $783 \mathrm{~cm}^{-1}$ can be attributed to $v(\mathrm{~V}-\mathrm{O}), \quad v(\mathrm{~W}=\mathrm{O})$, and $v(\mathrm{~W}-\mathrm{O}-\mathrm{W})$, respectively.

\section{Preparation and electrochemical measure of anode for LIBs}

$\left(\mathrm{NBu}_{4}\right)_{4}\left[\mathrm{VW}_{12} \mathrm{O}_{40}\right], \quad \mathrm{VW}_{12} @ \mathrm{MOCF}, \quad \mathrm{VW}_{12} @ \mathrm{MOCF}$ nanocrystals, and VW $12 @$ MOCF/SWNT-n composites $(n=1,2,3)$ were used as anode materials for rechargeable LIBs, respectively and the electrochemical measurements were carried out on coin-type cells. The working electrodes were prepared by mixing crystal materials, carbon black, and poly(vinylidene fluoride) (PVDF) at a weight ratio 7:2:1, which was passed on the $\mathrm{Cu}$ foils and followed by drying in vacuum at $120^{\circ} \mathrm{C}$ for 24 h. The cells were assembled in an argon-filled glovebox with the working electrode as-fabricated, metallic lithium foil as the counter electrode, and $1.0 \mathrm{M} \mathrm{LiPF}_{6}$ in ethylene carbonate/diethyl carbonate $(1: 1 \mathrm{v} / \mathrm{v})$ as the electrolyte. Battery capacity and galvanostatic charging/discharging test were carried out on a LAND CT2001A battery test system within a voltage range of $0-3.0 \mathrm{~V}$. The specific capacity was calculated based on the active material mass. Cyclic voltammetry (CV) and electrochemical impedance spectroscopy (EIS) were carried out on a CHI 660E electrochemical workstation. 

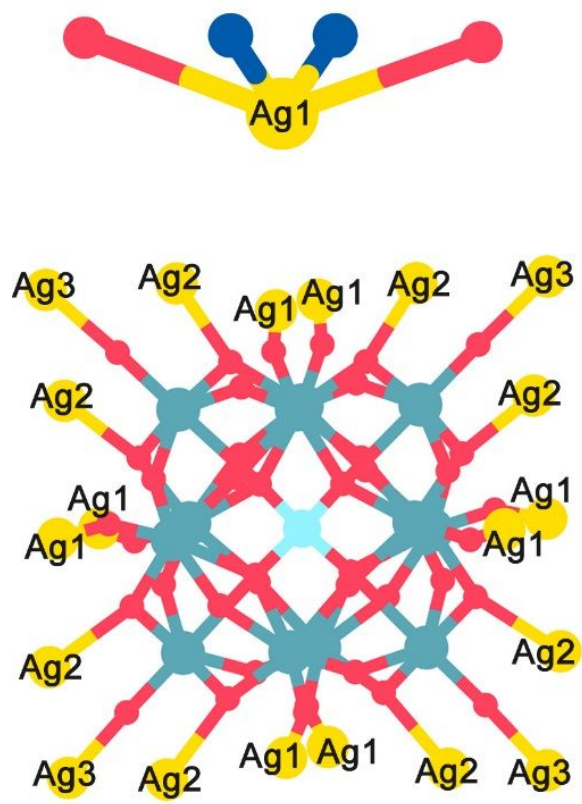
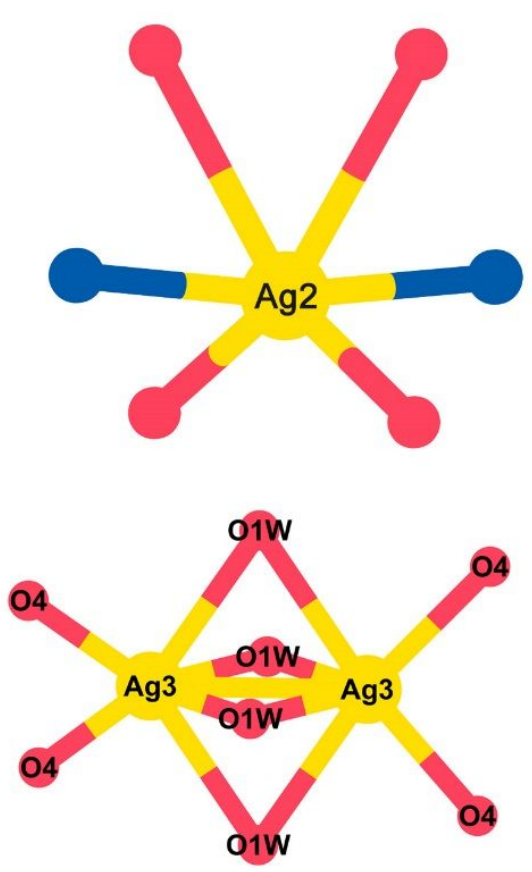

Fig. S2 Ball/stick view of the coordination mode of $\mathrm{Ag} 1, \mathrm{Ag} 2, \mathrm{Ag} 3$ and $\mathrm{VW}_{12}$ polyanion. $\mathrm{Ag}$ yellow spheres, $\mathrm{N}$, blue sphere, $\mathrm{O}$ red spheres, $\mathrm{V}$ cyan spheres, $\mathrm{W}$ teal blue spheres. All of the hydrogen atoms were omitted for clarity.

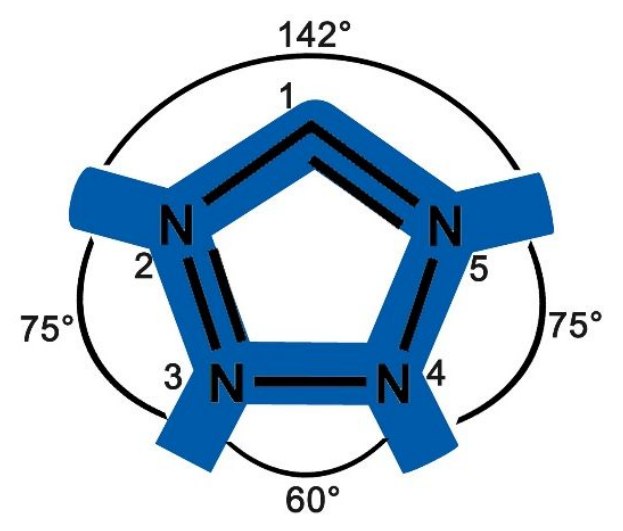

Fig. S3 The angular orientation of ttz ligand.

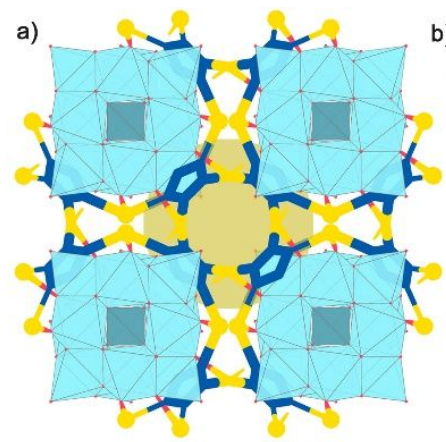

b)

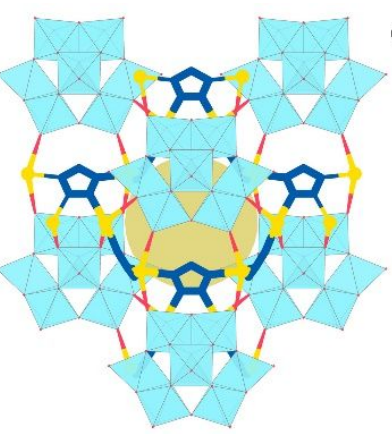

c)

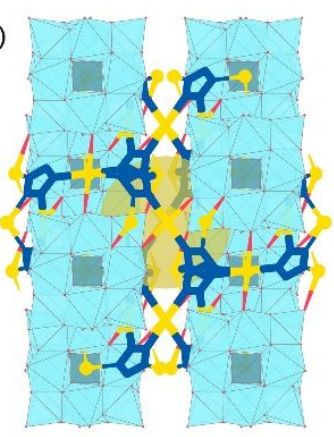

Fig. S4 $\mathrm{VW}_{12}$ polyanions as the external extrusion templates guide the assembly of nanocage $\mathrm{B}$ from different axial view. 


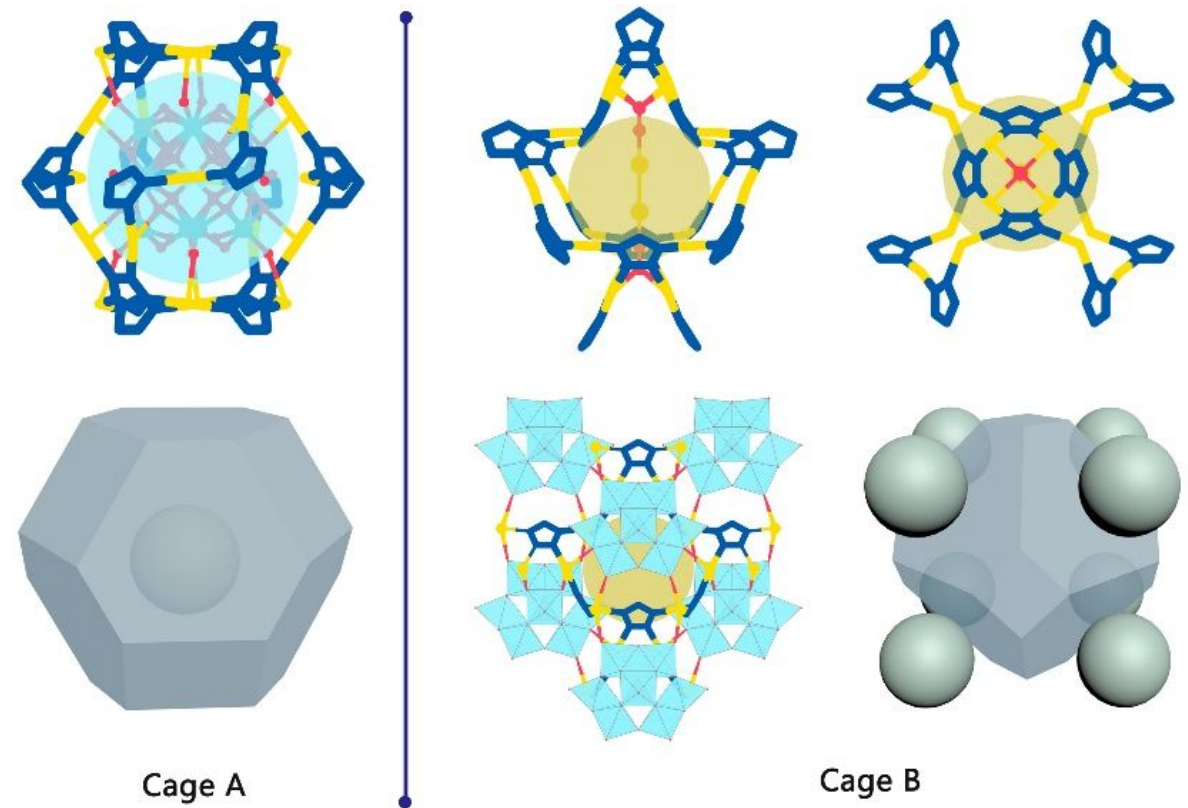

Fig. S5 Nanocage A and its inwardly occupied template of $\mathrm{VW}_{12}$ polyanions. The internal cavity of nanocage B is occupied by the disordered Ag3 ions and water clusters. And nanocage B is surrounded by eight $\mathrm{VW}_{12}$ polyanions.

a)

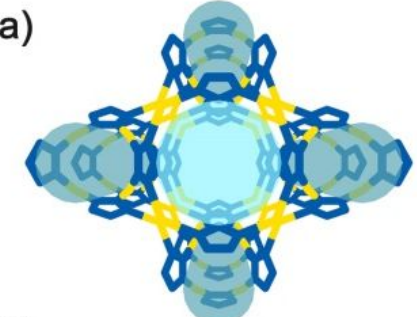

b)

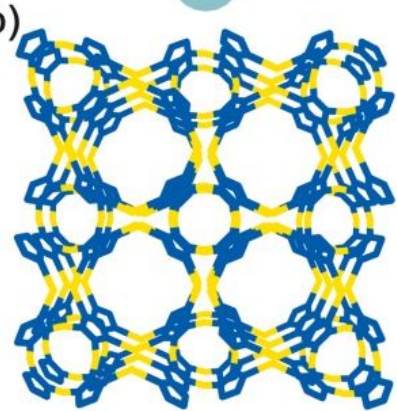

c)

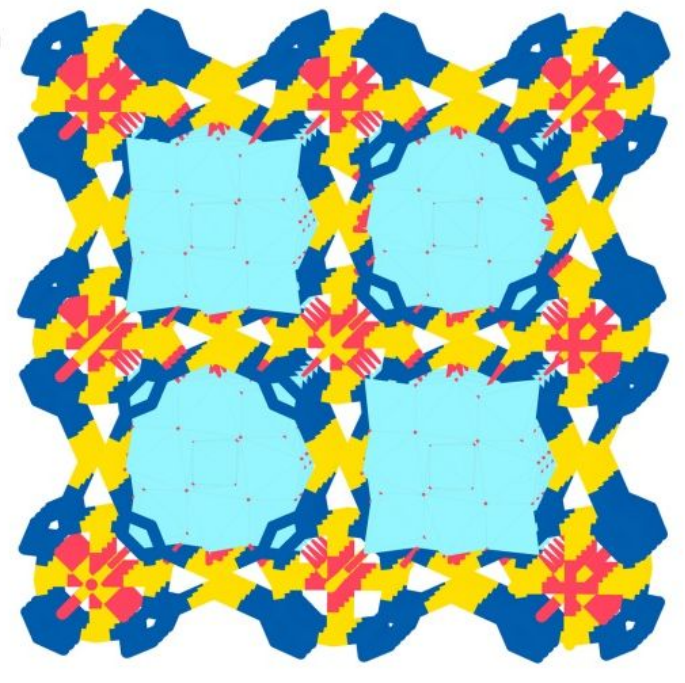

Fig. S6 a) Nanocage A and its surrounding nanocage B. b) The Ag-ttz framework consisting of nanocage A and B. c) The whole framework of VW $12 @$ MOCF. 


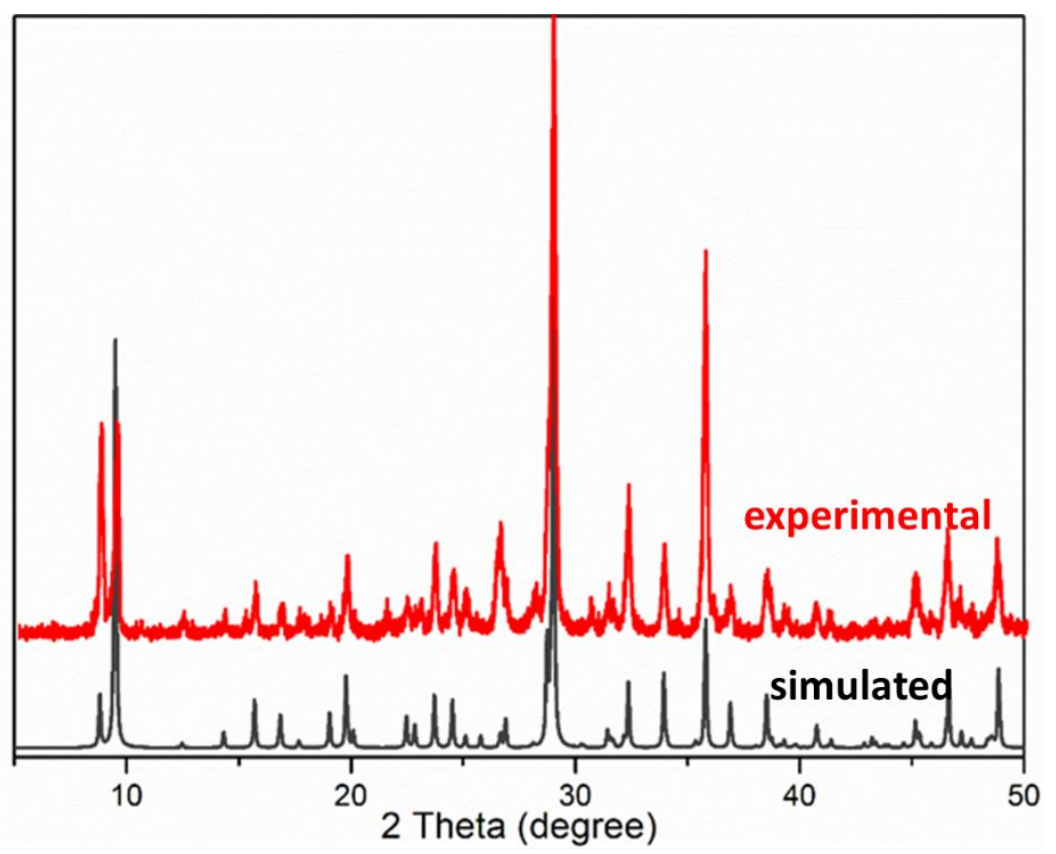

Fig. S7. The PXRD of VW $12 @ M O C F$

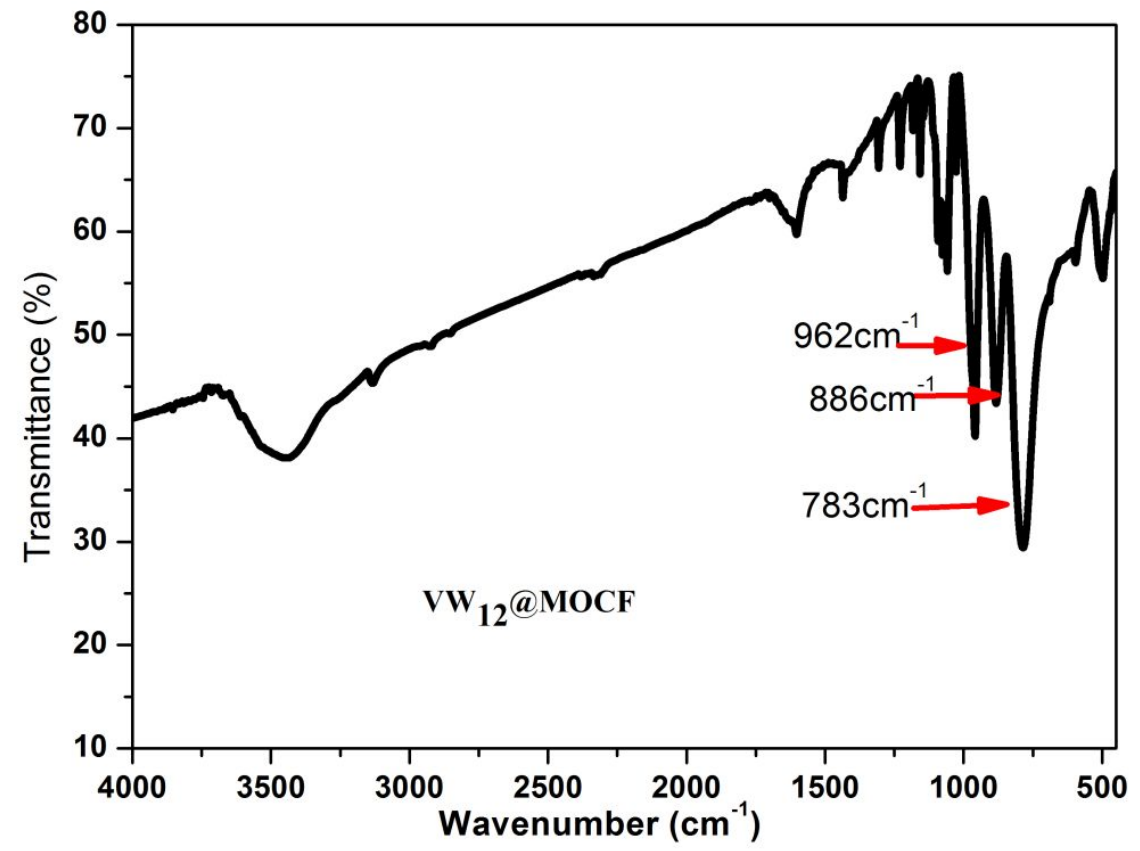

Fig. S8. The IR spectrum of $\mathrm{VW}_{12} @ \mathrm{MOCF}$, in which three characteristic bands at $c a .962,886$ and $783 \mathrm{~cm}^{-1}$ can be observed ascribed to the $v(\mathrm{~V}-\mathrm{O}), v(\mathrm{~W}=\mathrm{O})$, and $v(\mathrm{~W}-\mathrm{O}-\mathrm{W})$, respectively. 


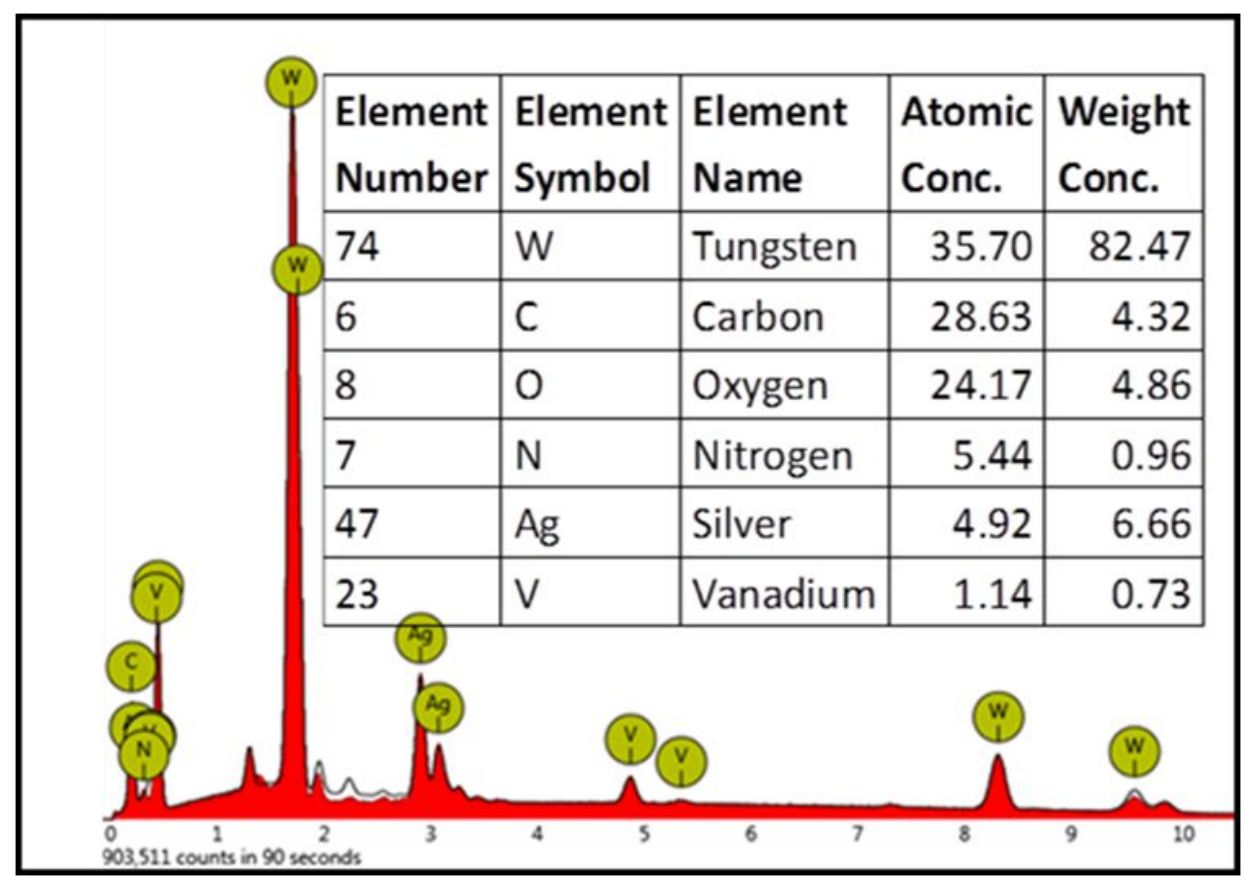

Fig. S9. The energy dispersive microscopy spectroscopy (EDS) of the $\mathrm{VW}_{12} @ \mathrm{MOCF}$ crystal.
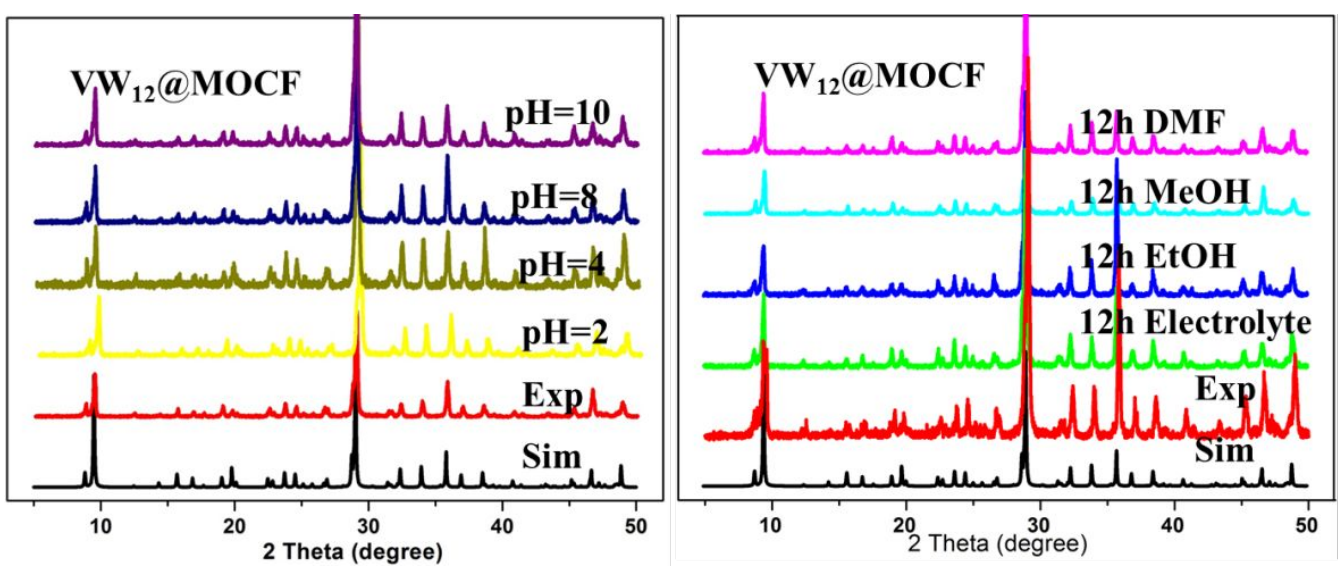

Fig. S10 PXRD patterns of VW $12 @$ MOCF soaked in aqueous solutions with different pH values at room temperature for $24 \mathrm{~h}$ and (b) immersed in electrolyte and other organic solvents at room temperature for 12h. "Sim": simulated pattern, and "Exp": as-synthesized sample. 


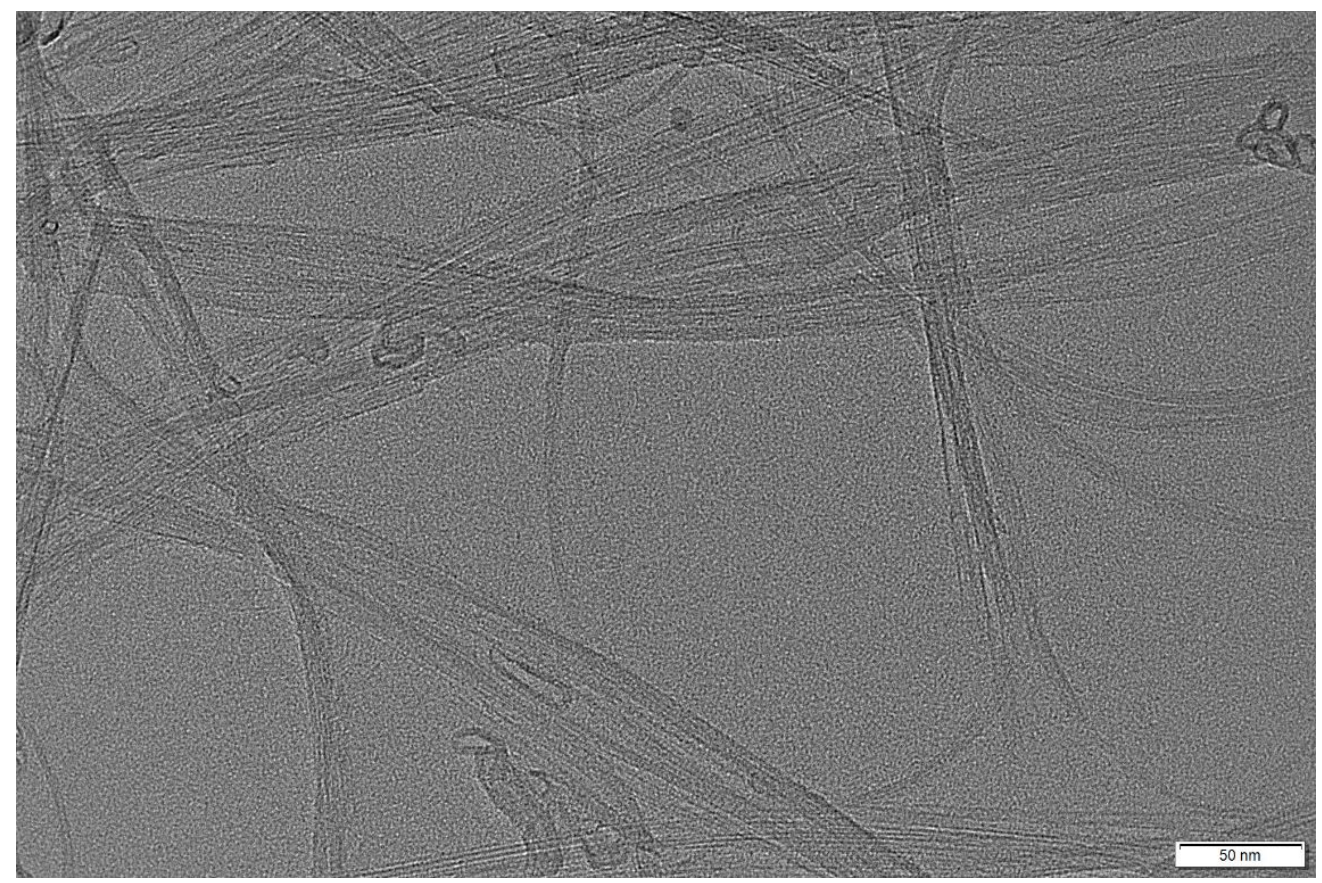

Fig. S11 TEM image of pristine SWNTs.

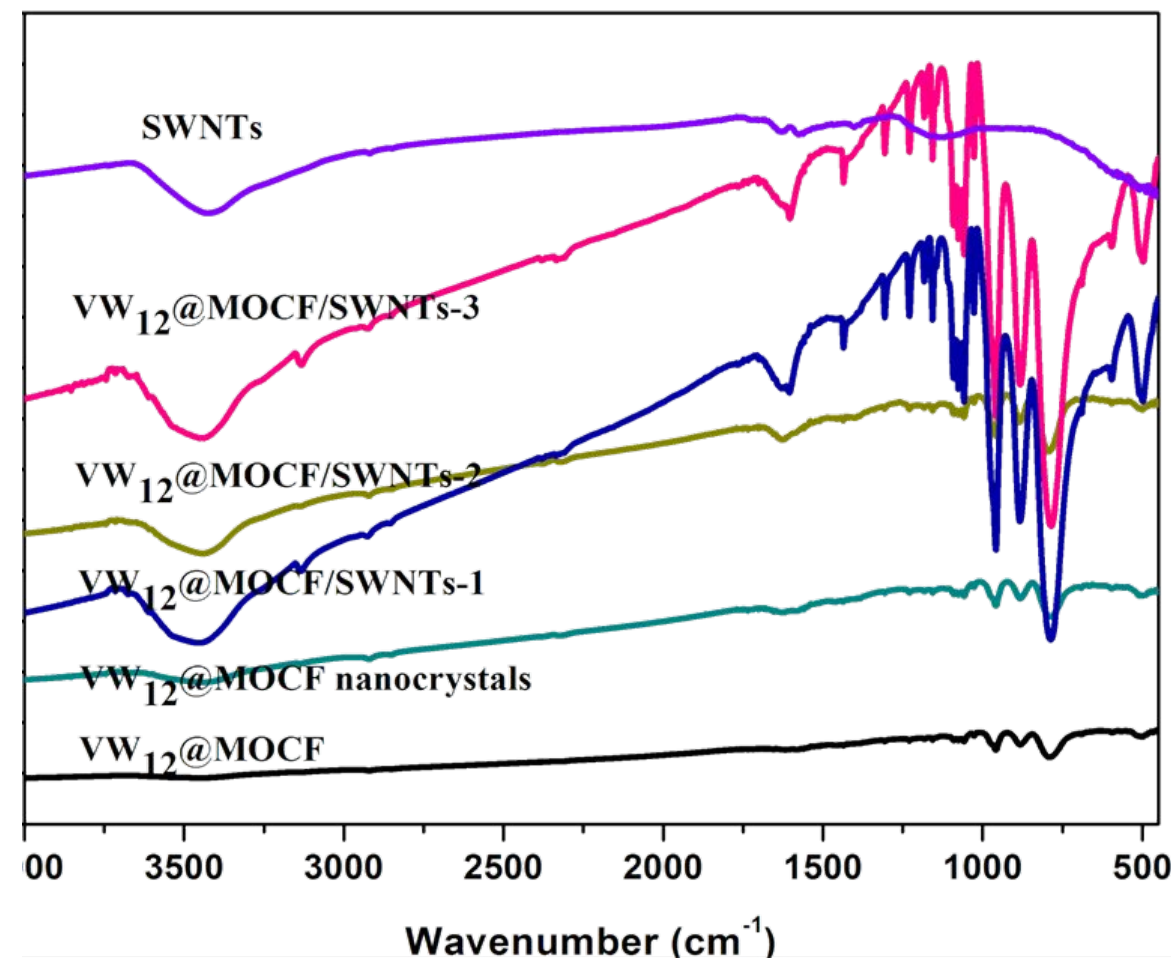

Fig. S12. IR spectra of the SWNT, VW $12 @ M O C F$ and VW $12 @ M O C F / S W N T-n$ nanocomposites. 

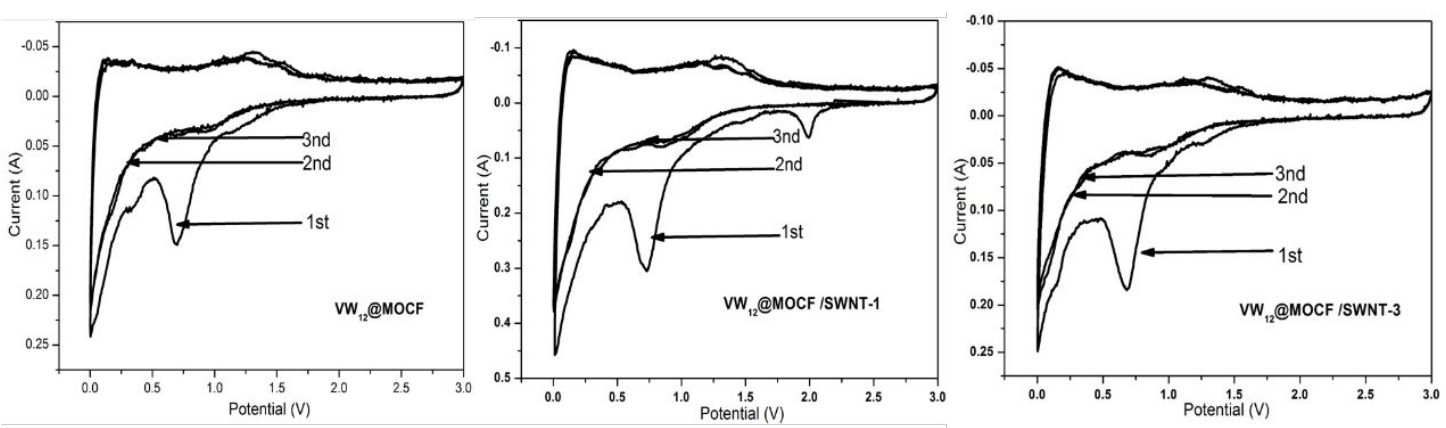

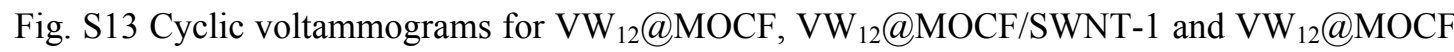
/SWNT-2 as anode materials at the range of $0-3 \mathrm{~V}$ (scan rate: $0.1 \mathrm{mV} \mathrm{s}^{-1}$ ).
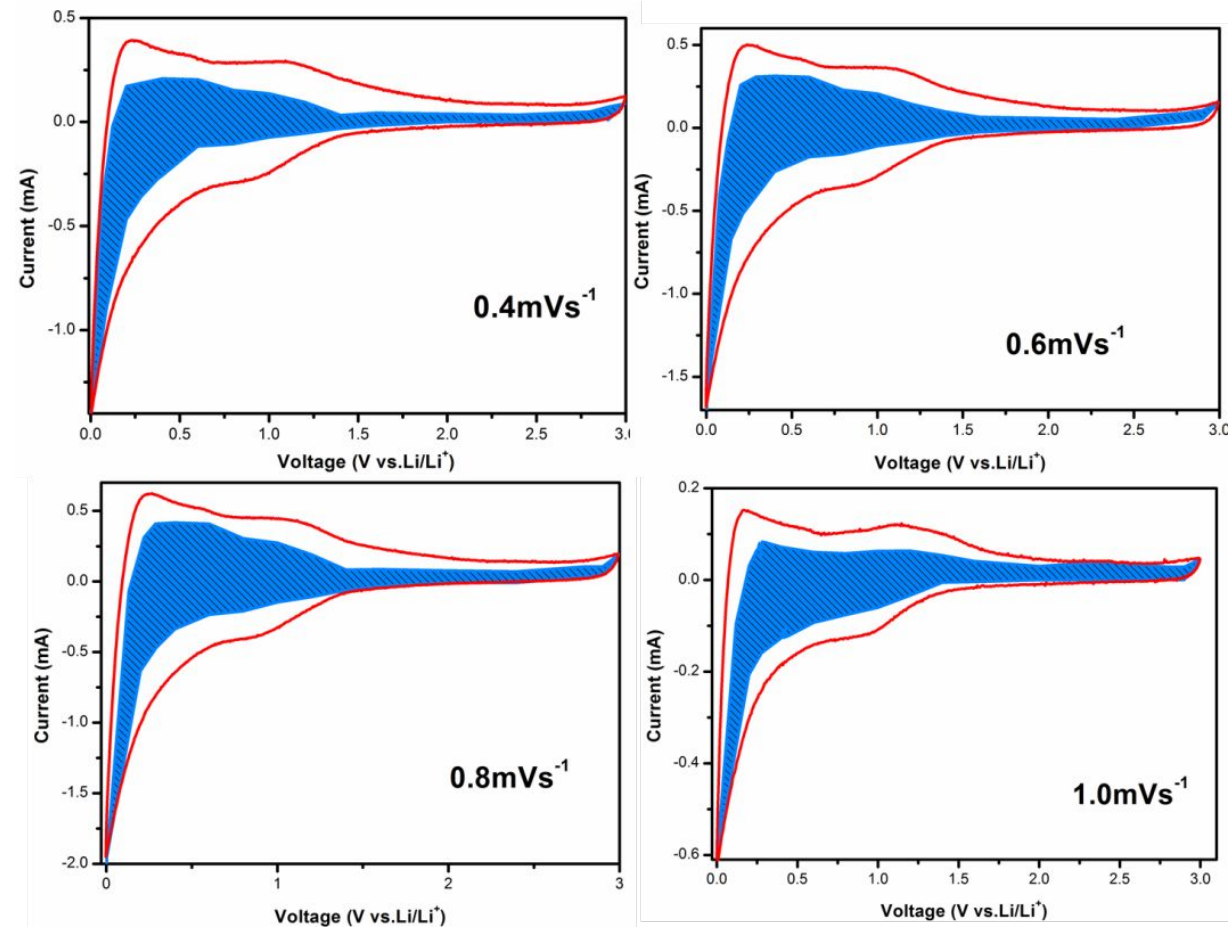

Fig. S14 Capacitive controlled charge storage contributions separated with cyclic voltammograms of fresh VW $\mathrm{VW}_{12} @ \mathrm{MOCF} / \mathrm{SWNT}-2$ at 0.4, 0.6, 0.8 and $1.0 \mathrm{mV} \mathrm{s}^{-1} \mathrm{scan}$. 
Table S1 Crystallographic data and structural refinement for VW ${ }_{12} @$ MOCF.

\begin{tabular}{|c|c|}
\hline Formula & $\mathrm{C}_{4} \mathrm{H}_{12} \mathrm{~N}_{16} \mathrm{Ag}_{10} \mathrm{VW}_{12} \mathrm{O}_{44}$ \\
\hline Fw & 4323.92 \\
\hline $\mathrm{T}(\mathrm{K})$ & $293(2)$ \\
\hline Crystal system & Tetranonal \\
\hline Space group & $\mathrm{I}-4 \mathrm{~m} 2$ \\
\hline $\mathrm{a}(\AA)$ & $14.170(5)$ \\
\hline$b(\AA)$ & $14.170(5)$ \\
\hline$c(\AA)$ & $12.279(5)$ \\
\hline$\alpha\left(^{\circ}\right)$ & 90 \\
\hline$\beta\left({ }^{\circ}\right)$ & 90 \\
\hline$\gamma\left({ }^{\circ}\right)$ & 90 \\
\hline$V\left(\hat{A}^{3}\right)$ & $2465.5(16)$ \\
\hline$Z$ & 2 \\
\hline $\operatorname{Dc}\left(\mathrm{g} \cdot \mathrm{cm}^{-3}\right)$ & 5.662 \\
\hline$\mu\left(\mathrm{mm}^{-1}\right)$ & 31.633 \\
\hline \multirow{2}{*}{ Reflections collected / unique } & $15555 / 8042$ \\
\hline & $/ \mathrm{R}(\mathrm{int})=0.0511$ \\
\hline Completeness to theta $=24.92$ & $99.7 \%$ \\
\hline $\mathrm{F}(000)$ & 3644 \\
\hline Data / restraints / parameters & 1187 / 63 / 113 \\
\hline finalR $1^{a}, w R 2^{b}[I>2 \sigma(I)]$ & $0.0262,0.0705$ \\
\hline finalR $1^{\mathrm{a}}, \mathrm{wR} 2^{\mathrm{b}}$ (all data) & $0.0263,0.0706$ \\
\hline GOF on $\mathrm{F}^{2}$ & 1.023 \\
\hline
\end{tabular}

$\left.{ }^{a} R_{1}=\Sigma\left(|| F_{0}|-| F_{c} \mid\right) / \Sigma\left|F_{0}\right|,{ }^{b} w R_{2}=\Sigma w\left(\left|F_{0}\right|^{2}-\left|F_{c}\right|^{2}\right)^{2} / \Sigma w\left(\left|F_{0}\right|^{2}\right)^{2}\right]^{1 / 2}$

Table S2 Selected bond lengths and angles for VW $\mathrm{VW}_{12} @ \mathrm{MOCF}$.

\begin{tabular}{cccc}
\hline bonds & Length $/ \AA$ & bonds & Length $/ \AA$ \\
\hline W1-O1 & $1.905(8)$ & Ag2O7 & $2.566(8)$ \\
W1-O21 & $1.898(3)$ & Ag2-N28 & $2.157(10)$ \\
W1-O5 & $1.713(8)$ & Ag2-N25 & $2.157(10)$ \\
W1-O62 & $1.914(5)$ & V1-O33 & $1.652(10)$ \\
W1-O32 & $2.322(7)$ & V1-O3 & $1.652(10)$ \\
W1-O7 & $1.914(7)$ & V1-O39 & $1.652(10)$ \\
\hline
\end{tabular}




\begin{tabular}{cccc}
\hline W2-O1 & $1.903(8)$ & $\mathrm{V} 1-\mathrm{O} 310$ & $1.652(10)$ \\
W2-O11 & $1.903(8)$ & $\mathrm{O} 4-\mathrm{Ag} 311$ & $2.409(11)$ \\
W2-O4 & $1.698(10)$ & $\mathrm{N} 2-\mathrm{N} 21$ & $1.323(19)$ \\
W2-O33 & $2.336(10)$ & $\mathrm{N} 2-\mathrm{N} 1$ & $1.328(14)$ \\
W2-O74 & $1.933(8)$ & $\mathrm{Ag} 2-\mathrm{O} 77$ & $2.566(8)$ \\
$\mathrm{W} 2-\mathrm{O} 73$ & $1.933(8)$ & $\mathrm{Ag} 3-\mathrm{Ag} 312$ & $2.719(9)$ \\
Ag1-N15 & $2.291(8)$ & $\mathrm{Ag} 3-\mathrm{O} 1 \mathrm{~W} 13$ & $2.59(2)$ \\
$\mathrm{Ag} 1-\mathrm{N} 1$ & $2.291(8)$ & $\mathrm{Ag} 3-\mathrm{O} 1 \mathrm{~W} 7$ & $2.59(2)$ \\
Ag2-Ag22 & $3.291(2)$ & $\mathrm{Ag} 3-\mathrm{O} 1 \mathrm{~W} 12$ & $2.59(2)$ \\
Ag2-Ag26 & $3.291(2)$ & $\mathrm{Ag} 3-\mathrm{O} 1 \mathrm{~W} 14$ & $2.59(2)$ \\
\hline
\end{tabular}

\begin{tabular}{cccc}
\hline Bond angle & Angle $^{\circ}$ & Bond angle & Angle $^{\circ}$ \\
\hline O21-W1-O1 & $85.8(4)$ & O3-V1-O39 & $109.7(4)$ \\
O5-W1-O1 & $101.4(3)$ & O33-V1-O3 & $109.7(4)$ \\
O5-W1-O21 & $101.4(4)$ & O33-V1-O39 & $109.1(7)$ \\
O62-W1-O1 & $158.4(4)$ & O38-V1-O33 & $109.7(4)$ \\
O62-W1-O21 & $89.2(4)$ & O38-V1-O3 & $109.1(7)$ \\
O62-W1-O5 & $100.2(4)$ & W21-O1-W1 & $150.4(4)$ \\
O32-W1-O1 & $85.0(3)$ & W1-O2-W11 & $152.1(6)$ \\
O32-W1-O21 & $85.6(4)$ & Ag310-O4-W2 & $153.3(6)$ \\
O32-W1-O5 & $170.7(3)$ & W12-O6-W1 & $121.0(6)$ \\
O32-W1-O62 & $73.7(3)$ & W1-O3-W12 & $91.7(4)$ \\
O7-W1-O1 & $87.8(3)$ & W29-O3-W12 & $92.6(3)$ \\
O7-W1-O21 & $158.1(4)$ & W29-O3-W1 & $92.6(3)$ \\
O7-W1-O5 & $100.4(3)$ & V13-O3-W12 & $124.0(3)$ \\
O7-W1-O62 & $89.1(4)$ & V13-O3-W1 & $124.0(3)$ \\
O7-W1-O32 & $72.9(3)$ & V13-O3-W29 & $123.0(5)$ \\
O11-W2-O1 & $87.3(5)$ & W24-O7-W1 & $122.2(4)$ \\
O4-W2-O1 & $102.2(4)$ & Ag26-O7-W1 & $107.9(3)$ \\
O4-W2-O11 & $102.2(4)$ & Ag26-O7W-24 & $119.7(3)$ \\
O33-W2-O1 & $85.3(3)$ & N21-N2Ag-25 & $117.1(3)$ \\
O33-W2-O11 & $85.3(3)$ & N1-N2-Ag25 & $132.6(7)$ \\
O33-W2-O4 & $169.6(5)$ & N2-N1-Ag15 & $124.5(7)$ \\
O74-W2-O1 & $88.3(3)$ & C11-N1-Ag15 & $127.6(8)$ \\
O74-W2-O11 & $157.4(3)$ & C11-N1-N2 & $105.7(9)$ \\
O73-W2-O1 & $157.4(3)$ & N11-C1-N1 & $109.6(13)$ \\
\hline
\end{tabular}




\begin{tabular}{cccc}
\hline Bond angle & Angle $^{\circ}$ & Bond angle & Angle $/^{\circ}$ \\
\hline O73-W2-O11 & $88.3(3)$ & O4-Ag3-O410 & $104.2(5)$ \\
O74-W2-O4 & $100.4(4)$ & Ag311-Ag3-O410 & $127.9(3)$ \\
O73-W2-O4 & $100.4(4)$ & Ag311-Ag3-O4 & $127.9(3)$ \\
O73-W2-O33 & $72.3(3)$ & O1W6-Ag3-O4 & $81.2(3)$ \\
O74-W2-O33 & $72.3(3)$ & O1W12-Ag3-O410 & $81.2(3)$ \\
O73-W2-O74 & $87.3(4)$ & O1W11-Ag3-O4 & $142.85(11)$ \\
N1-Ag1-N15 & $131.2(4)$ & O1W11-Ag3-O410 & $81.2(3)$ \\
O76-Ag2-Ag22 & $141.27(17)$ & O1W13-Ag3-O4 & $81.2(3)$ \\
O7-Ag2-Ag22 & $83.03(17)$ & O1W6-Ag3-O410 & $142.85(11)$ \\
O7-Ag2-O76 & $124.5(3)$ & O1W13-Ag3-O410 & $142.85(11)$ \\
N25-Ag2-Ag22 & $62.9(3)$ & O1W12-Ag3-O4 & $142.85(11)$ \\
N27-Ag2-Ag22 & $110.2(3)$ & O1W6-Ag3-O1W13 & $74.1(4)$ \\
N27-Ag2-O76 & $100.3(3)$ & O1W12-Ag3-O1W11 & $74.1(4)$ \\
N25-Ag2-O7 & $100.3(3)$ & O1W6-Ag3-O1W11 & $74.1(4)$ \\
N25-Ag2-O76 & $83.9(3)$ & O1W12-Ag3-O1W6 & $116.8(5)$ \\
N27-Ag2-O7 & $83.9(3)$ & O1W12-Ag3-O1W13 & $74.1(4)$ \\
N27-Ag2-N25 & $171.0(5)$ & O1W13-Ag3-O1W11 & $116.8(5)$ \\
O38-V1-O39 & $109.7(4)$ & Ag3-O1W-Ag313 & $63.2(6)$ \\
\hline
\end{tabular}

Symmetry transformations used to generate equivalent atoms:

${ }^{1},+\mathrm{Y},+\mathrm{Z} ;{ }^{2}+\mathrm{X},-\mathrm{Y},+\mathrm{Z} ;{ }^{3}-\mathrm{Y},+\mathrm{X}, 1-\mathrm{Z} ;{ }^{4}+\mathrm{Y},+\mathrm{X}, 1-\mathrm{Z} ;{ }^{5}+\mathrm{Y},+\mathrm{X},-\mathrm{Z} ;{ }^{6} 1-\mathrm{X},+\mathrm{Y},+\mathrm{Z} ;{ }^{7} 1 / 2-\mathrm{Y}, 1 / 2-\mathrm{X}, 1 / 2-\mathrm{Z} ;{ }^{8} 1 /$

2-X,1/2-Y,1/2+Z; ${ }^{9}-\mathrm{X},-\mathrm{Y},+\mathrm{Z} ;{ }^{10}+\mathrm{Y},-\mathrm{X}, 1-\mathrm{Z} ;{ }^{11}-\mathrm{X}, 1-\mathrm{Y},+\mathrm{Z} ;{ }^{12} 1 / 2-\mathrm{Y}, 1 / 2+\mathrm{X}, 1 / 2-\mathrm{Z} ;{ }^{13}-1 / 2+\mathrm{Y}, 1 / 2-\mathrm{X}, 1 / 2$

$-Z ;{ }^{14}-1 / 2+Y, 1 / 2+X, 1 / 2-Z$

Table S3 Structural summary of POMs-templated MOFs nanocage containing

Ag-N/C bonds

\begin{tabular}{|l|l|l|l|}
\hline Ligands & Ag-N/C & POMs-based MOFs & Ref. \\
\hline & $\begin{array}{c}\text { 24-nuclear } \\
{\left[\mathrm{Ag}_{24}(\mathrm{trz})_{18}\right]} \\
\text { nanocages }\end{array}$ & 1 \\
\hline oxylic acid
\end{tabular}




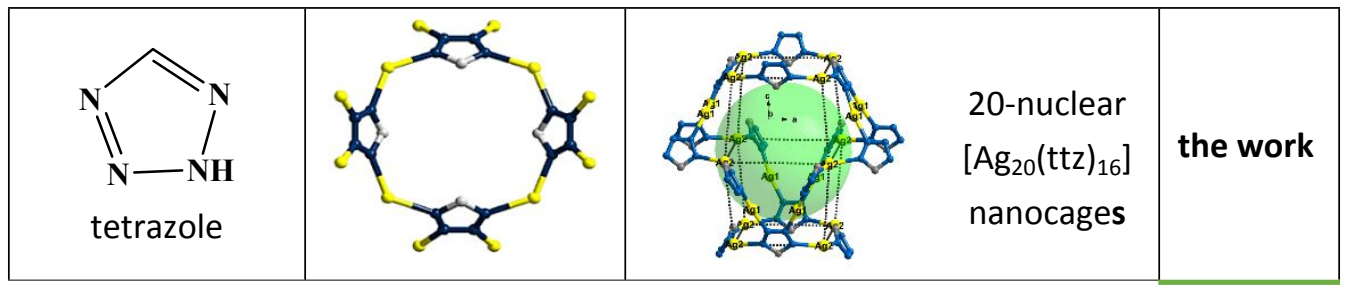

\section{References}

(1) Sha, J. Q.; Yang, X. Y.; Chen, Y. Y.; Zhu, P. P.; Song, Y. F., Jiang, J. Z. Fabrication and Electrochemical Performance of PolyoxometalateBased Three-Dimensional Metal Organic Frameworks Containing Carbene Nanocages, ACS Appl. Mater. Interfaces 2018, 10, 16660-16665.

(2) Sha, J. Q.; Li, M. T.; Yang, X. Y.; Sheng, N.; Li, J. S.; Zhu, M. L.; Liu, G. D.; Jiang. J. Z. New Route toward POM[6]Catenane Members for Lithium-Ion Batteries. Cryst. Growth Des. 2017, 17, 3775-3782. 